\title{
OPTIMIZACIÓN DEL PROCESO DE EXTRACCIÓN DE LOS FRUCTOOLIGOSACÁRIDOS DE YACÓN (Smallantus sonchifolius)
}

\author{
Inga Guevara, Marianela ${ }^{\mathrm{a}-}$; Betalleluz Pallardel, Indira ${ }^{\mathrm{a}-}$; Kina Noborikawa, Melissa ${ }^{\mathrm{a}-}$; \\ Campos Gutierrez, David ${ }^{\text {a* }}$
}

\begin{abstract}
RESUMEN
La investigación comprendió dos etapas: En la primera se realizó un screennig o cribado para evaluar el efecto de los factores más significativos: temperatura, tiempo y relación materia prima/solvente (MP:S) sobre el proceso de extracción de fructooligosácaridos (FOS) y azúcares reductores (AR) a partir de harina de yacón usándose un arreglo factorial $2^{\mathrm{K}}$. Los niveles planteados para los factores fueron: tiempo 5-40 minutos; relación MP:S 1:10-1:70 g/ $\mathrm{ml}$; temperatura $30-90^{\circ} \mathrm{C}$; las variables respuestas fueron: rendimiento de extracción de FOS y AR. Los resultados indicaron que el factor relación MP:S tuvo un efecto significativo sobre la extracción de FOS y AR ( $p$-value $<0,05)$. En la siguiente etapa se evaluó diferentes niveles de relación MP:S (1:10-1:100 g/ml) manteniendo el tiempo 5 minutos y la temperatura $30^{\circ} \mathrm{C}$. La máxima extracción y la mayor recuperación del extracto se obtuvo con la relación MP:S 1:30 g/ml. Finalmente, se empleo dos modelos cinéticos para describir el proceso de extracción de FOS y AR (1-60 min ), dando valores de $\mathrm{R}^{2-:}$ 0,97-0,99, respectivamente. Los resultados señalan que la relación MP:S 1:30, tiempo 10 minutos y temperatura de $30{ }^{\circ} \mathrm{C}$ aseguran un máximo rendimiento en la extracción de FOS (37,60 $\pm 0,50 \%$ bs).
\end{abstract}

Palabras clave: Extracción, fructooligosacáridos, yacón, azúcares reductores

\section{OPTIMIZING THE EXTRACTION PROCESS FRUCTOOLIGOSACARIDOS OF YACON (Smallantus sonchifolius)}

\begin{abstract}
ABSTRAC
The research involved two stages: In the first one, screening methodology was performed to evaluate the effect of the most significant factors: temperature, time and ratio raw material: solvent (MP: S) on the extraction of fructooligosaccharides (FOS) and reducing sugars (AR) from yacon flour. A factorial arrangement $2 \mathrm{~K}$ were used; the levels for the factors were: time 5-40 minutes; ratio MP: S 1:10-1:70 g / ml; temperature 30-90 ${ }^{\circ} \mathrm{C}$; responses variables were extraction yield of FOS and AR. The results indicated that only the factor ratio MP: $\mathrm{S}$ had significant effect on the extraction of FOS and AR (p-value $<0.05$ ). In the next stage, many levels of ratio MP: $\mathrm{S}(1: 10$ to $1: 100 \mathrm{~g} / \mathrm{ml})$ were evaluated holding for time 5 minutes and temperature at $30^{\circ} \mathrm{C}$. Maximum extraction and greatest recovery of the extract was obtained with ratio MP:S 1:30. Finally, two kinetic models were used to describe the extraction process of FOS and AR (1-60 min), values of $\mathrm{R}^{2}: 0,97$ to 0,99 were obtained respectively. The results indicate that the ratio MP: S 1:30, time 10 minutes and temperature $30{ }^{\circ} \mathrm{C}$ assure maximum performance in the extraction of FOS $(37,60 \pm 0,50 \% \mathrm{bs})$.
\end{abstract}

Key words: Extraction, fructooligosaccharides, yacon, reducing sugars

(a) Instituto de Biotecnología (IBT), Universidad Nacional Agraria La Molina. Av La Molina s/n La Molina. Lima-Perú. (*) autor de correspondencia: dcampos@lamolina.edu.pe 


\section{INTRODUCCIÓN}

La extracción es el primer paso para aislar y caracterizar compuestos de interés, debiéndose esta operación mantener la integridad del compuesto y evitar la co-extracción de otros compuestos que interfieran en su aprovechamiento. Las técnicas utilizadas para la extracción emplean de forma muy general una etapa de molienda o trituración, seguida de la exposición en solventes con aplicación de calor.

Los FOS son carbohidratos que consisten en una cadena de unidades de fructosa con una unidad de glucosa terminal unidas por enlace glucosídico $\beta(2 \longrightarrow 1)$, característica que los define como oligosacáridos no digeribles, que hace que no puedan ser degradados por las enzimas digestivas humanas ${ }^{1}$. Por otro lado, debido a que el consumo de FOS no eleva el nivel de glucosa en la sangre ${ }^{2}$ puede ser incluido en la dieta de los diabéticos ${ }^{3}$. Industrialmente los FOS se obtienen a partir de la hidrólisis de la inulina, un polisacárido que está presente en cantidades importantes en las raíces de la achicoria o por procesos sintéticos a partir de la sacarosa, la cual es sometida a transfructosilación con $\beta$-fructofuranosidasa ${ }^{4}$. Es por ello, que se presenta como una alternativa la obtención de FOS a partir de las raíces de yacón, en las cuales están presentes en forma natural y en cantidades apreciables ${ }^{5}$.

Si bien es cierto existen estudios previos sobre la extracción de FOS a partir de yacón, éstos se realizan en estado fresco; además no se ha concretado aún una metodología con parámetros y condiciones que evidencien obtener la máxima recuperación del compuesto funcional.

\section{PARTE EXPERIMENTAL}

Materia prima: Las raíces de yacón adquiridas del mercado fueron cortadas en rodajas de 5 $\mathrm{mm}$ de espesor, sometidas a un blanqueado en agua a ebullición por 6 minutos y secadas a $65^{\circ} \mathrm{C}$ hasta alcanzar un contenido de una humedad de 3,5\%; luego fueron molidas en molino de laboratorio (Marca IKA, Modelo A11 Basic) y la harina obtenida se tamizó utilizando una malla $\mathrm{N}^{\circ} 20$ (833 micras).

\section{Métodos de análisis}

Azúcares reductores: Se usó el método del ácido dinitrosalicílico ${ }^{6}$.

Contenido de fructooligosacáridos: Se realizó el procedimiento señalado por Jaime et al. ${ }^{7}$ con algunas modificaciones. Se utilizó HPLC-IR (Waters Associates, modelo 2695); detector de índice de refracción Waters Associates (Modelo 2414) y columna Shodex Asahipak NH2P-40 3E (4,6 mm ID x $250 \mathrm{~mm}$ ) y pre-columna Shodex NH2P-50G 3A (4,6 mm ID x 10 $\mathrm{mm})$; el solvente de elución fue acetonitrilo: agua $(80: 20)$ a un flujo de $0,5 \mathrm{ml} / \mathrm{min}$ y $42^{\circ} \mathrm{C}$; durante 20 minutos. La cantidad de muestra inyectada fue $20 \mu \mathrm{l}$. La muestra, estándares (glucosa, fructosa y sacarosa) y la fase móvil se filtraron a través de un filtro de $0,22 \mu \mathrm{m}$ PVDF Millipore ${ }^{\circledR}$ antes de la inyección.

\section{Metodología experimental}

Preparación del extracto: La harina se pesó y se añadió agua destilada con una relación materia prima/solvente $1 / 250 \mathrm{ml}$; los matraces fueron llevados a baño maría con agitación constante a diferentes temperaturas y tiempos de extracción según el diseño experimental. 
Luego se filtró a vacío, utilizando papel Watman de paso lento $\mathrm{N}^{\circ} 1$, para la obtención del extracto bruto, en los cuales se determinó el contenido de frutooligósacaridos (FOS) y azúcares reductores (AR). Los resultados se expresaron en g/100 de materia seca.

\section{Diseño experimental y análisis estadístico}

El estudio se realizó en dos etapas. La primera consistió en un "screening" para identificar los principales factores que tienen efecto significativo en la extracción de FOS, y la segunda etapa, una optimización de las condiciones de extracción, considerando los factores seleccionados de la primera.

\section{Etapa screening o cribado}

Los tres factores evaluados en el proceso de extracción de FOS fueron: relación materia prima/solvente $(\mathrm{X} 1)$, temperatura $(\mathrm{X} 2)$ y tiempo(X3). Los niveles estudiados se presentan en la tabla 1. El efecto de cada una de estos factores y de sus interacciones, fue evaluado utilizando un diseño factorial de $2^{\mathrm{K}}$ con tres puntos centrales.

Tabla 1. Factores y sus niveles considerados en el arreglo factorial $2 \mathrm{k}$ aplicado en la etapa de screening para la extracción de FOS

\begin{tabular}{lccc}
\hline \multirow{2}{*}{ Factores } & Código & \multicolumn{2}{c}{ Niveles } \\
\cline { 3 - 4 } & & -1 & 1 \\
\hline Relación MP:S $(\mathrm{g} / \mathrm{ml})$ & $\mathrm{X} 1$ & $1: 10$ & $1: 70$ \\
Temperatura $\left({ }^{\circ} \mathrm{C}\right)$ & $\mathrm{X} 2$ & 30 & 90 \\
Tiempo $(\mathrm{min})$ & $\mathrm{X} 3$ & 5 & 40 \\
\hline
\end{tabular}

Una matriz de 8 corridas fue generada del diseño factorial. La función respuesta investigada (variable dependiente) correspondió al rendimiento en la extracción de FOS (Y, \%). El análisis estadístico se realizó con el software Statgraphics Centurion XV (Stat Point Inc., VA, USA).

\section{Etapa de optimización de la extracción de FOS}

Identificados en la etapa de screening los factores significativos, se investigó el efecto de la relación MP:S y la cinética de la extracción de FOS y AR de acuerdo a lo siguiente:

Efecto de la relación MP: S en la extracción de FOS y AR: Para poder determinar el nivel MP:S que maximiza la extracción se ensayó diferentes relaciones $(1: 10,1: 20,1: 30,1: 40$, $1: 50,1: 70,1: 90$ y $1: 100 \mathrm{~g} / \mathrm{ml})$, manteniendo constante el tiempo y la temperatura del proceso en los niveles establecidos en la primera etapa.

Cinética de extracción de FOS y AR: Para evaluar la cinética de extracción de FOS y AR de la harina de yacón se empleó dos modelos matemáticos (tabla 2) que simulen este proceso con el menor error en las estimaciones. 
Tabla 2. Modelos matemáticos aplicados al proceso de extracción de azúcares

\begin{tabular}{|c|c|c|c|}
\hline Modelo & Ecuación & Parámetros del Modelo & Referencia \\
\hline $\begin{array}{l}\text { Ley de la } \\
\text { velocidad } \\
\text { (Ley de } \\
\text { Fick) }\end{array}$ & $\begin{aligned} C t & =\frac{C e^{2} K t}{1+C e k t} \\
C t & =\frac{t}{\left(\frac{1}{h}\right)+\left(\frac{t}{C e}\right)} \\
h & =k C e^{2}\end{aligned}$ & $\begin{array}{l}\text { Ct: Concentración del carbohidrato* a un } \\
\text { tiempo } \mathrm{t}(\mathrm{g} / \mathrm{l}) \\
\text { Ce: Concentración de equilibrio del } \\
\text { carbohidrato* }(\mathrm{g} / \mathrm{l}) \\
\mathrm{K}: \quad \text { Tasa de extracción }(\mathrm{g} / \mathrm{l} \mathrm{min}) \\
\mathrm{h}: \quad \text { Tasa inicial de extracción }(\mathrm{g} / \mathrm{l} \mathrm{min})\end{array}$ & 8 \\
\hline $\begin{array}{l}\text { Ley de Fick } \\
\text { modificada }\end{array}$ & $\left.\frac{C o-C}{C o}=\left(1-\mathrm{b}^{\prime}\right) e^{-K t}\right)$ & $\begin{array}{l}\text { Co: Concentración del carbohidrato* en la } \\
\text { matriz sólida }(\mathrm{g} / 100 \mathrm{~g} \mathrm{~ms}) \\
\mathrm{C} \text { : Concentración del carbohidrato* } \\
\text { remante en la matriz sólida }(\mathrm{g} / 100 \mathrm{~g} \mathrm{~ms}) \text {. } \\
\mathrm{b}^{\prime}: \text { Coeficiente de extracción cinética en } \\
\text { la etapa de lavado } \\
\mathrm{K} \text { : Coeficiente de extracción cinético en } \\
\text { la etapa de difusión }\left(\mathrm{min}^{-1}\right)\end{array}$ & 9 \\
\hline
\end{tabular}

(*) Carbohidratos: Azúcares reductores (AR), fructooligosacáridos (FOS)

\section{Caracterización de la harina de yacón}

\section{RESULTADOS Y DISCUSIÓN}

La tabla 3 muestra la composición de la harina en azúcares y FOS. La harina posee 96,45\% de sólidos totales, formado en su mayoría por FOS, azúcares reductores y sacarosa con contenidos de $37,9 \%, 33,14 \%, 7,11 \%$, respectivamente; valores menores a los reportados por Lobo et al. ${ }^{10}$ en harina de yacón procedente de Sao Paulo (Brasil) con 55,3\% de FOS como el principal componente de almacenamiento y $8,9,13,5$ y $13,4 \%$ para glucosa, fructosa y sacarosa, respectivamente.

Estas variaciones puede deberse a diferencias en el estado de madurez y zona geográfica de procedencia como concluyó Chirinos ${ }^{11}$. Otro aspecto a considerar es la perecibilidad de esta raíz; estudios han demostrado que luego de la cosecha las raíces empiezan un rápido proceso de cambio en la composición química: los FOS son hidrolizados en azúcares simples por la acción de la enzima fructanohidrolasa, que los convierte en fructosa, sacarosa y glucosa. La forma de almacenamiento es determinante ${ }^{12}$. Se reporta que después de una semana de almacenamiento a temperatura ambiente alrededor del 30 al $40 \%$ los FOS se transformaron en azúcares simples; otro estudio indica la conveniencia del almacenamiento bajo temperatura de refrigeración para hacer lenta esta velocidad de conversión13. Una de las razones que limita una industrialización de los FOS de yacón es el contenido de azúcares simples que lo acompañan y que puede incrementarse en la postcosecha; estos azúcares suelen acompañar a la extracción del prebiótico y determina la necesidad de operaciones de purificación posterior. 
Tabla 3. Composición de la harina de yacón en FOS y azúcares (*)

\begin{tabular}{|c|c|}
\hline Componente & $\begin{array}{c}\text { Contenido } \\
(\%)\end{array}$ \\
\hline FOS (b.s.) & $37,9 \pm 0,51$ \\
\hline Azúcares reductores (b.s.) & $33,14 \pm 0,03$ \\
\hline Sacarosa (b.s.) & $7,11 \pm 0,25$ \\
\hline Humedad (\%) & $3,55 \pm 0,11$ \\
\hline
\end{tabular}

(*) Promedio de tres repeticiones

\section{Primera etapa de optimización: Screening}

El factor relación MP:S resultó tener efecto significativo en el rendimiento de extracción de FOS y AR $(\mathrm{p}<0,05)$ tal como se puede observar en el diagrama de efectos estandarizados de Pareto (figura 1a y 1b), respectivamente. Los factores temperatura, tiempo, al igual que sus interacciones no tuvieron influencia significativa.

Investigaciones que estudian la extracción de FOS e inulina de otras fuentes señalan que la temperatura es un factor determinante; sin embargo, los resultados obtenidos demuestran lo contrario en los niveles evaluados $\left(30-90^{\circ} \mathrm{C}\right)$. Paseephol et al. ${ }^{14}$ recomiendan la extracción con agua en ebullición por 10-15 minutos para alcachofa de la var. Jerusalén. Toneli et al. ${ }^{15}$ emplearon agua a $80^{\circ} \mathrm{C}$ por una hora con agitación para la extracción de inulina de raíces seca de achicoria La diferencia puede deberse a que en el caso del yacón las moléculas son de bajo peso molecular (FOS); mientras que en la alcachofa y achicoria se tiene oligofructosas de alto peso molecular (inulina) cuya solubilidad se incrementa marcadamente con la temperatura, siendo casi insoluble a $25{ }^{\circ} \mathrm{C}$ y alcanzando una solubilidad aprox de $35 \%$ (peso/volumen) a $90^{\circ} \mathrm{C}^{16}$.
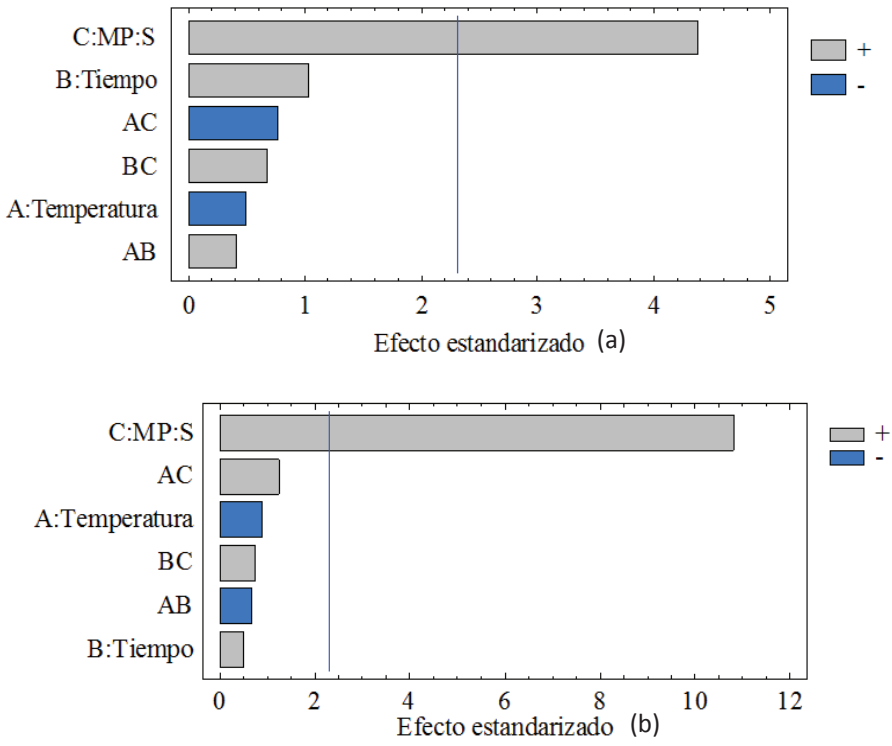

Figura 1. Diagrama de Pareto estandarizado del rendimiento de extracción de FOS (a) y AR (b). 
Adicionalmente, Apolinário et al. ${ }^{17}$ indican la mayor solubilidad de los FOS comparados con la inulina debido a las cadenas más cortas de sus moléculas. En el caso de los AR se observa similar comportamiento debido a que tienen una fuerte interacción con las moléculas de agua por su carácter altamente hidrofílico ${ }^{18}$.

Teniendo en cuenta criterios de ahorro de energía y tiempo de la operación se seleccionó una temperatura de $30^{\circ} \mathrm{C}$ y un tiempo de 5 minutos para continuar con la siguiente etapa, condiciones bajo las cuales se extrae el máximo contenido de FOS.

\section{Segunda etapa: Etapa de optimización}

\section{Efecto de la relación MP:S en la extracción de FOS y AR}

La tabla 4 muestra el efecto de los niveles de la relación MP:S sobre la extracción de FOS y AR a partir de harina de yacón. Se observa una recuperación significativamente $(\mathrm{p}<0.05)$ de FOS cuando la relacion MP:S va de 1:10 a 1:20; relaciones mayores presentan un ligero incremento en la extración que no son estadísticamente significativas. A mayor proporción de solvente el gradiente de concentración durante la difusión del FOS del interior al solvente se ve incrementado.

Tabla 4. Efecto de la relación MP:S en la extracción de FOS yAR durante 5 minutos a 30 ${ }^{\circ} \mathrm{C}$ a partir de harina de yacón $(*)$

\begin{tabular}{ccc}
\hline $\begin{array}{c}\text { Relación MP:S } \\
(\mathrm{g} / \mathrm{ml})\end{array}$ & $\begin{array}{c}\text { FOS } \\
(\%, \mathrm{bs})\end{array}$ & $\begin{array}{c}\text { Azúcares reductores } \\
(\%, \mathrm{bs})\end{array}$ \\
\hline $1: 10$ & $34,46 \pm 0,99 \mathrm{a}$ & $25,78 \pm 0,65 \mathrm{a}$ \\
$1: 20$ & $36,64 \pm 0,49 \mathrm{c}$ & $33,49 \pm 0,77 \mathrm{~b}$ \\
$1: 30$ & $37,60 \pm 0,50 \mathrm{c}$ & $33,46 \pm 0,54 \mathrm{~b}$ \\
$1: 40$ & $36,40 \pm 0,90 \mathrm{c}$ & $33,47 \pm 0,10 \mathrm{~b}$ \\
$1: 50$ & $37,77 \pm 0,86 \mathrm{c}$ & $33,90 \pm 0,45 \mathrm{~b}$ \\
$1: 70$ & $37,14 \pm 1,08 \mathrm{c}$ & $33,99 \pm 0,50 \mathrm{~b}$ \\
$1: 90$ & $34,97 \pm 0,23 \mathrm{~b}$ & $33,92 \pm 0,19 \mathrm{~b}$ \\
$1: 100$ & $32,08 \pm 0,24 \mathrm{a}$ & $33,10 \pm 0,46 \mathrm{~b}$ \\
\hline (*)Promedio de tres repeticiones, $\mathrm{n}=3 \pm$ desviación estándar; letras diferentes dentro de una columna \\
indican diferencias significativas $\mathrm{p}<0,05$.
\end{tabular}

Teniendo en cuenta esta consideración y habiendo observado que a relaciones bajas también no hay una buena recuperación del extracto del residuo sólido durante la filtración, se recomienda una relación MP:S de 1:30. Chirinos ${ }^{11}$ menciona que es importante determinar la relación MP:S óptima durante el proceso de extracción de oligofructanos, ya que el solvente tiene la particularidad de permitir obtener un sistema fluido que favorezca la llegada del solvente hacia las célula y favorecer la salida de los componentes que en ellas se encuentra.

\section{Cinética de extracción de FOS y AR}

La tabla 5 muestra los parámetros de los modelos de cinética aplicados al proceso de extracción; los valores de h, Ce y $\mathrm{K}$ fueron obtenidos de la pendiente e intercepto al graficar 
t/Ct versus $t$ (Ley de velocidad). La tasa de extracción inicial (h) de FOS es mayor que la de AR, lo que indica una mayor difusividad de los FOS. Los valores obtenidos pueden ser comparados con otros procesos de extracción en los cuales se aplica temperatura para obtener un mayor rendimiento, como los obtenidos en la extracción de compuestos antioxidantes de la granadilla cuando se aplica temperaturas por encima de $60{ }^{\circ} \mathrm{C}$ (h varía entre $35-100,7$ $\mathrm{g} / \mathrm{l} \mathrm{min})^{8}$, mientras que para el yacón la temperatura no tiene efecto. Con el modelo de Fick modificado se halló b y k, parámetros que indican una mayor difusividad de los FOS frente a los $\mathrm{AR}$ en las etapas de extracción de la harina de yacón. Los altos coeficientes de determinación $\left(\mathrm{R}^{2}=0,97-0,99\right)$ señalan que la extracción de FOS y AR se ajustan adecuadamente a los dos modelos empleados.

Tabla 5. Parámetros de los modelos de cinética aplicados al proceso de a extracción de FOS y AR a una relación MP:S 1:30 g/ml y $30{ }^{\circ} \mathrm{C}$.

\begin{tabular}{|c|c|c|c|c|}
\hline Azúcares & $\begin{array}{c}\text { Parámetros estimados } \\
\text { (1) }\end{array}$ & $\begin{array}{c}\text { Parámetros estimados } \\
\text { (2) }\end{array}$ & $\begin{array}{l}\mathrm{R}^{2} \\
(1)\end{array}$ & $\begin{array}{l}\mathrm{R}^{2} \\
(2)\end{array}$ \\
\hline FOS & $\begin{array}{l}\mathrm{h}=40.317(\mathrm{~g} / \mathrm{L} \min ) \\
\mathrm{K}=0.246(\mathrm{~g} / \mathrm{L} \min ) \\
\mathrm{Ce}: 12.84(\mathrm{~g} / \mathrm{L})\end{array}$ & $\begin{array}{l}\mathrm{b}=0,8128 \\
\mathrm{k}=0,2485 \mathrm{~min}^{-1}\end{array}$ & 0,99 & 0,98 \\
\hline AR & $\begin{array}{l}\mathrm{h}=43.290(\mathrm{~g} / \mathrm{L} \min ) \\
\mathrm{K}=0.363(\mathrm{~g} / \mathrm{L} \mathrm{min}) \\
\mathrm{Ce}: 10.917(\mathrm{~g} / \mathrm{L})\end{array}$ & $\begin{array}{l}\mathrm{b}=0,7955 \\
\mathrm{k}=0,206 \mathrm{~min}^{-1}\end{array}$ & 0,99 & 0,97 \\
\hline
\end{tabular}

(1) Modelo Ley de la velocidad (Ley de Fick) (2) Modelo Ley de Fick modificado

En las figuras 2a, 2b, 3a y 3b se muestra las cinéticas de extracción de FOS y AR, bajo los parámetros seleccionados en las etapas previas (temperatura: $30{ }^{\circ} \mathrm{C}$, relación MP:S: 1:30 g/ $\mathrm{ml}$ ), se observa la tendencia típica de este tipo de operación; la cantidad extraída de FOS se incrementa rápidamente y luego alcanza un periodo constante de extracción. Este incremento rápido ocurre dentro de los primeros 5 minutos. Esta tendencia es explicada por Chun et al. ${ }^{9}$ quienes señalan que la curva de extracción con solvente está compuesta típicamente de una etapa de extracción rápida (etapa de lavado) y una etapa de extracción lenta (etapa difusión). El mecanismo de extracción empieza cuando las moléculas del solvente penetran en la matriz vegetal, causando que la capa del citoplasma sea expuesta directamente al solvente19. Esto facilita la disolución de los compuestos activos en el solvente. En el inicio del proceso de extracción, la etapa rápida corresponde a velocidad constante de extracción.

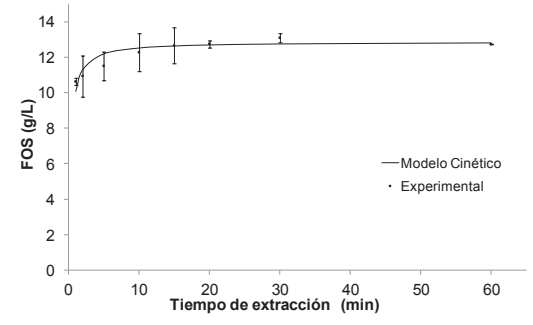

(a)

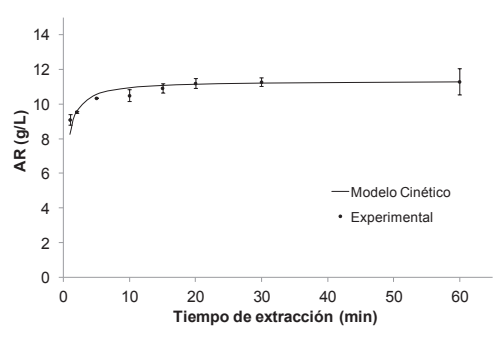

(b)

Figura 2. Cinética de extracción de FOS (a) y AR (b) a una relación MP:S 1:30 g/ml y a $30{ }^{\circ} \mathrm{C}$. Modelo Ley de la velocidad (Ley de Fick). 


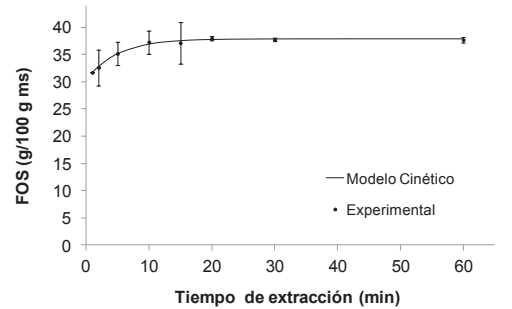

(a)

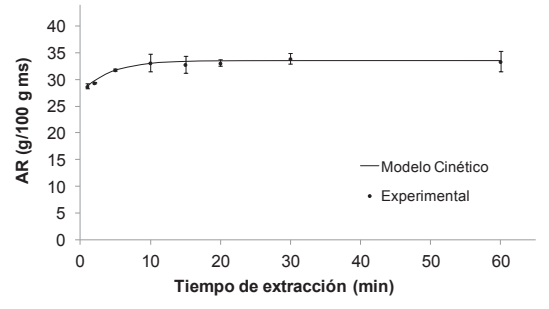

(b)

Figura 3. Cinética de extracción de FOS (a) y AR (b) a una relación MP:S 1:30 g/ml y a $30{ }^{\circ} \mathrm{C}$. Modelo Ley de Fick modificado

\section{CONCLUSIONES}

Se encontró que el factor relación MP/S influye significativamente en el proceso de extracción de FOS de harina de yacón, a partir de la relación MP:S, 1:30 se asegura la máxima extracción de FOS. Los factores temperatura y tiempo resultaron no ser significativos en el proceso. Los mejores resultados se obtuvieron a temperatura ambiente, durante 10 minutos y la relación materia prima/solvente 1:30. Los modelos cinéticos la Ley de velocidad y la Ley de Fick modificado simulan adecuadamente el proceso de extracción de FOS Y AR de la harina de yacón.

La extracción de FOS de la harina de yacón también trae consigo la extracción de otros compuestos solubles que pueden encontrarse en el extracto, como polisacáridos, azúcares reductores y sacarosa, además de compuestos fenólicos, enzimas etc., que le dan turbidez y color al extracto, por lo que resulta necesario estudiar la clarificación y purificación del extracto preservando el contenido de FOS.

\section{AGRADECIMIENTO}

Este proyecto ha sido financiado por el Fondo para la Innovación, Ciencia y Tecnología (FINCYT-CONTRATO: 142-FINCyT-IA-2013).

\section{BIBLIOGRAFÍA}

1. Sabater, M. Efectos de las poliaminas y los fructooligosacáridos de la dieta sobre la maduración intestinal en cerdos destetados precozmente. Memoria presentada para optar el grado de Doctora en Biología. Universidad de Murcia, España. 2008.

2. Luo J., Van Yperselle M., Rizkalla S., Rossi F., Bornet F., Slama G. Chronic consumption of short-chain fructooligosaccharides does not affect basal hepatic 30 Jarabe de yacón: Principios y procesamiento glucose production or insulin resistance in type 2 diabetics. Journal of Nutrition, 2000; 130: 1572-1577.

3. Manrique I., Párraga A., Hermann M. Jarabe de yacón: Principios y procesamiento. Centro Internacional de la Papa (CIP). Lima, Perú. 2005. 
4. Madrigal L., Sangronis E. La inulina y derivados como ingredientes claves en alimentos funcionales. Archivos Latinoamericanos De Nutrición, 2007; 57(4): 387 - 396

5. Lachman J., Fernandez E.C., Orsák M. Yacon [Smallanthus sonchifolius (Poepp. Et Endl.) H. Robinson] chemical composition and use - a review. Plant Soil Environ, 2003; 49: 283-290.

6. Miller, G.. Use of dinitrosalicylic acid reagent for determination of reducing sugar. Analytical Chemistry, 1959; 31: 426-428.

7. Jaime L., Martín-Cabrejas M.M., Mollá E., López-Andreú F. Effect of storage on fructan and fructoloigosaccharide of onion (Allium cepa L.). J. Agric. Food Chem, 2001; 49: 982-988.

8. Wenjuan Q., Zhongli P., Haile M. Extraction modeling and activities of antioxidants from pomegranate marc. Journal of Food Engineering, 2010; 99: 16-23.

9. Chung-Hung Ch., Rozita Y., Gek-Cheng N. Modeling and kinetics study of conventional and assisted batch solvent extraction. Chemical engineering research and design, 2014; 9(2):1169-1186.

10. Lobo A., Colli C., Alvares E., Filisetti, T. Effects of fructans-containing yacon (Smallanthus sonchifolius Poepp\&Endl.) flour on caecum mucosal morphometry, calcium and magnesium balance, and bone calcium retention in growing rats. British Journal of Nutrition, 2007; 97: 776-785.

11. Chirinos R. Obtención y caracterización de los oligofructanos a partir de la raíz del yacón (Smallanthus sonchifolia Poepp. \&Endl.). Tesis para optar el grado de Magister Scientiae. Universidad Nacional Agraria La Molina. Lima, Perú 1999.

12. Graefe S., Hermann M., Manrique I., Golombek S., Buerkert A. Effects of post-harvest treatments on the carbohydrate composition of yacon roots in the Peruvian Andes. Field Crops Res, 2004; 86: 157-165.

13. Asami T., Minamisawa K., Tsuchiya T., Kano K., Hori I., Ohyama T., Kubota M., Tsukihashi T. Fluctuation of oligofructan contents in tubers of yacon (Polymnia sonchifolia) during growth and storage. Japanese Journal of Soil Science and Plant Nutrition, 1991; 62: 621-627.

14. Paseephol T., Small D., Sherkat F. Process optimization for fractionating Jerusalem artichoke fructans with ethanol using response surface methodology. Food Chemistry, 2007; 104(1): 73-80.

15. Toneli J. T. C. L., Park K. J., Murr F. E. X., Negreiros A. A. Effect of moisture on the 
272 Inga Guevara, Marianela; Betalleluz Pallardel, Indira; Kina Noborikawa, Melissa, Campos Gutierrez, David

microestructure of inulin powder. Ciência e Tecnologia de Alimentos, 2008; 28 (1): 122131.

16. Dobre T., Stroescu M., Stoica A., Draghici E., Antohe N. Inulin Extraction and Encapsulation. Rev Chimie, 2008; 53(67): 215-217.

17. Apolinário A.C., Goulart de Lima B.P., Esberard de Macedo N., Pessoa A., Converti A., Alexsandro da Silva. Inulin-type fructans: A review on different aspects of biochemical and pharmaceutical technology. Carbohydrate Polymers, 2014; 101: 368 - 378.

18. Maia M.C.A., Cal-Vidal J. Kinetics of water uptake by citrus juices in powder form. Int J Food Sci Technol., 1994; 29(2): 137-141.

19. Crossley J.I., Aguilera J.M. Modeling the effect of microstructure on food extraction. Journal Food Process. Eng., 2001; 24: 161 - 177 